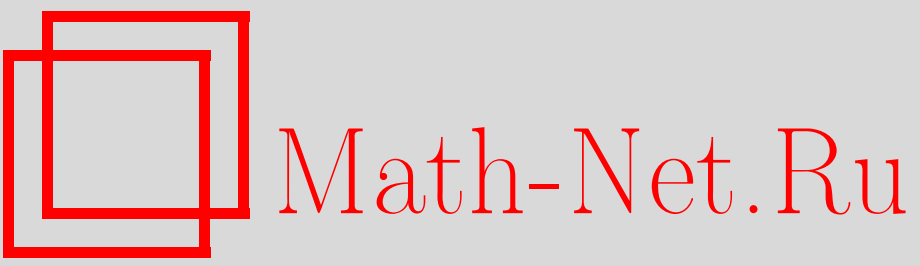

В. Л. Верещагин, Асимптотические разложения для частных решений шестого уравнения Пенлеве, ТМФ, 2001, том 128, номер 2, 193-204

DOI: https://doi.org/10.4213/tmf491

Использование Общероссийского математического портала Math-Net.Ru подразумевает, что вы прочитали и согласны с пользовательским соглашением

http://www . mathnet.ru/rus/agreement

Параметры загрузки:

IP : 18.234 .197 .8

26 апреля 2023 г., 11:16:18 
ТЕОРЕТИЧЕСКАЯ

И МАТЕМАТИЧЕСКАЯ

ФИЗИКА

Том 128, № 2

август, 2001

(C) 2001 г.

\author{
В. Л. Верещагин*
}

\title{
АСИМПТОТИЧЕСКИЕ РАЗЛОЖЕНИЯ ДЛЯ ЧАСТНЫХ РЕШЕНИЙ ШЕСТОГО УРАВНЕНИЯ ПЕНЛЕВЕ
}

\begin{abstract}
Развит формализм метода усреднения для уравнений Пенлеве, в частности для шестого уравнения. Поставлена задача описания асимптотического поведения шестого трансцендента Пенлеве при условии стремления модуля независимой переменной $\mathrm{k}$ бесконечности. Соответствующие разложения имеют в главном члене эллиптическую функцию (анзац), чьи параметры зависят от переменной из-за уравнения модуляций. Для шестого уравнения Пенлеве явно выписаны эллиптический анзац и уравнение модуляций. Найдено частное решение уравнения модуляций, дающее не известное ранее асимптотическое разложение для частного решения шестого уравнения Пенлеве.
\end{abstract}

\section{1. ВВЕДЕНИЕ}

В середине 19-го века в число наиболее насущных задач анализа входили исследование и классификация обыкновенных дифференциальных уравнений. Методы для такого исследования были разработаны Фуксом и Пуанкаре и основываются на изучении особенностей обших решений. Так, имея дело с линейным уравнением, мы можем получить нужную информацию об особенностях общего решения из вида коэффициентов уравнения. Ситуация коренным образом меняется, когда в качестве объекта изучения взяты нелинейные дифференциальные уравнения. Теперь для определения положения особенности и ее типа недостаточно знать вид коэффициентов уравнения, так как в общем случае характеристики особых точек зависят от начальных данных. Нам интересна зависимость особенностей от уравнения, а не от начальных данных, поэтому естественным выглядит желание ограничить рассмотрение лишь теми нелинейными уравнениями, чьи решения имеют критические точки, не зависящие от начальных условий (как это было для всех линейных уравнений).

ОПрЕДЕЛЕниЕ. Точка, в которой решение уравнения имеет особенность, называется подвижной (неподвижной), если ее положение зависит (не зависит) от начальных данных этого решения.

${ }^{*}$ Институт математики с вычислительным центром УрО РАН, Уфа, Россия. E-mail: vadim@imat.rb.ru 
ОПРЕДЕЛЕНИЕ. Точка называется критической точкой дифференциального уравнения, если общее решение неоднозначно в любой ее окрестности.

Для построения классификации дифференциальных уравнений естественно считать эквивалентными уравнения, переводимые друг в друга дробно-рациональными преобразованиями. Таким образом Фукс, Пуанкаре и позже Пенлеве сформулировали и решали следующую задачу: описать все классы эквивалентности нелинейных дифференциальных уравнений вида

$$
\Omega\left(x, y, y^{\prime}, \ldots, y^{(n)}\right)=0
$$

где $\Omega$ - некоторая функция без подвижных критических точек относительно групп преобразований вида

$$
\tilde{x}=\varphi(x), \quad \tilde{y}=\frac{\alpha(x) y+\beta(x)}{\gamma(x) y+\delta(x)}
$$

где $\alpha, \beta, \gamma, \delta, \varphi \in \mathbf{C}$ - голоморфные функции.

Классификация всех алгебраических уравнений (1) первого порядка с алгебраической функцией $\Omega$ и $n=1$ была получена Фуксом и Пуанкаре: каждое алгебраическое дифференциальное уравнение первого порядка без подвижных критических точек эквивалентно одному из следующих двух уравнений:

$$
\left(\frac{d y}{d x}\right)^{2}=4 y^{3}-g_{2} y-g_{3}, \quad g_{2}, g_{3} \in \mathbf{C},
$$

- уравнение на ю-функцию Вейерштрасса и

$$
\frac{d y}{d x}=a(x) y^{2}+b(x) y+c(x)
$$

- уравнение Риккати, где $a, b, c$ - голоморфные функции.

Многочисленные попытки построить подобную классификацию для уравнений второго порядка $(n=2)$ были безуспешными, до того как Пенлеве ограничил рассмотрение рациональными уравнениями вида

$$
\frac{d^{2} y}{d x^{2}}=R\left(x, y, y^{\prime}\right)
$$

с фиксированными критическими точками. Результатом огромного количества вычислений, проведенных Пенлеве и его учениками, явился список из 50 классов эквивалентности. Из этих 50 уравнений лишь 6 имеют обшие решения, не представимые в терминах известных спецфункций. Решения упомянутых шести дифференциальных уравнений являются новыми функциями и по праву носят название трансцендентов Пенлеве (см. [1]). В последние годы традиционный интерес к этим уравнениям как объекту классической теории дифференциальных уравнений был значительно усилен появлением их многочисленных приложений в различных областях науки. 
Главным объектом рассмотрения в данной работе является шестое уравнение (PVI) этого семейства

$$
\begin{aligned}
y^{\prime \prime}= & \frac{1}{2}\left(\frac{1}{y}+\frac{1}{y-1}+\frac{1}{y-x}\right)\left(y^{\prime}\right)^{2}-\left(\frac{1}{x}+\frac{1}{x-1}+\frac{1}{y-x}\right) y^{\prime}+ \\
& +\frac{y(y-1)(y-x)}{x^{2}(x-1)^{2}}\left(\alpha+\beta \frac{x}{y^{2}}+\gamma \frac{x-1}{(y-1)^{2}}+\delta \frac{x(x-1)}{(y-x)^{2}}\right),
\end{aligned}
$$

где греческие буквы обозначают независимые параметры. Цель работы - исследовать асимптотические свойства шестого трансцендента $y(x)$ для больших значений $|x|$.

\section{2. ИЗОМОНОДРОМНЫЕ И ИЗОСПЕКТРАЛЬНЫЕ СИСТЕМЫ}

Одним из важнейших свойств уравнений Пенлеве является свойство изомонодромности.

Рассмотрим проколотую комплексную сферу $\mathbf{C P}^{1} \backslash\left\{t_{1}, \ldots, t_{2 g+1}, t_{2 g+2}=\infty\right\}$ и $2 g+1(2 \times 2)$-матриц $A_{j}$. Тогда представление фундаментальной группы $\pi_{1}\left(\mathbf{C P}^{1}\right.$ $\left.\left\{t_{1}, \ldots, t_{2 g+1}, \infty\right\}\right)$ может быть получено с помощью решений системы Фукса

$$
\frac{d Y(\lambda)}{d \lambda}=\sum_{j=1}^{2 g+1} \frac{A_{j}}{\lambda-t_{j}} Y(\lambda)
$$

на $(2 \times 2)$-матрицы $Y(\lambda)$. Зафиксируем начальную точку $t_{0} \in \mathbf{C P}^{1} \backslash\left\{t_{1}, \ldots, t_{2 g+1}, \infty\right\}$ и традиционным образом определим $\pi_{1}$ как множество петель $\gamma=\gamma(t), 0 \leqslant t \leqslant 1$, $\gamma(0)=\gamma(1)=t_{0}$. Возьмем невырожденную матрицу $Y_{0}$ и предположим, что $Y(\lambda)-$ решение (7) такое, что $Y\left(t_{0}\right)=Y_{0}$. Для каждого $\gamma \in \pi_{1}$ положим

$$
M(\gamma)=M\left(\gamma ; t_{0}, Y_{0}\right) \equiv Y_{0}^{-1} Y(\gamma(1)),
$$

где $Y(\gamma(1))$ обозначает аналитическое продолжение решения $Y(\lambda)$ вдоль петли $\gamma, t_{0}=$ $\gamma(0) \mapsto \gamma(1)=t_{0}$. Тогда простое вычисление показывает, что отображение

$$
\pi_{1} \ni \gamma \mapsto M(\gamma)
$$

задает линейное представление $\pi_{1}$, которое называется монодромным представлением для системы (7). Соответствуюшая данным $A_{j}, t_{j}$ подгруппа $M(\gamma), \gamma \in \pi_{1}$, называется группой монодромии системы Фукса (7). Все эти группы изоморфны по отношению к сопряжению, т.е. $M\left(\gamma ; t_{0}, Y_{0}\right)=Y_{0}^{-1} M\left(\gamma ; t_{0}, Y_{0}\right) Y_{0}$, поэтому под группой монодромии следует понимать соответствуюший класс эквивалентности по отношению к сопряжению.

Шлезингер [2] поставил следующий вопрос: каким образом матрицы $A_{j}$ должны зависеть от величин $t_{i}$, для того чтобы элементы описанной выше группы монодромии были интегралами системы (7)? Ответ на него таков: матричные коэффициенты $A_{j}$ должны удовлетворять системе нелинейных дифференциальных уравнений

$$
\frac{\partial A_{i}}{\partial t_{j}}=\left(1-\delta_{i j}\right) \frac{\left[A_{i}, A_{j}\right]}{t_{i}-t_{j}}-\delta_{i j} \sum_{k \neq i} \frac{\left[A_{i}, A_{k}\right]}{t_{i}-t_{k}},
$$


называемых уравнениями Шлезингера. Таким образом, система (8) является условием изомонодромности (изомонодромных деформаций) для системы линейных уравнений $(7)$.

Несколько позже Гарнье [3] сделал следующее наблюдение. Рассмотрим систему (8), в правой части которой переменные величины $t_{j}$ заменены на постоянные параметры $T_{j}$. Такое автономное уравнение

$$
\frac{\partial A_{i}}{\partial t_{j}}=\left(1-\delta_{i j}\right) \frac{\left[A_{i}, A_{j}\right]}{T_{i}-T_{j}}-\delta_{i j} \sum_{k \neq i} \frac{\left[A_{i}, A_{k}\right]}{T_{i}-T_{k}}
$$

определяет изоспектральные деформации для $M(\lambda)$, т.е. многочлен $\operatorname{det}(M(\lambda)-\mu I)$ инвариантен вдоль потока $t$. Риманова поверхность $(\lambda, \mu)$, задаваемая характеристическим уравнением $\operatorname{det}(M(\lambda)-\mu I)=0$, называется спектральной кривой. Гарнье также доказал интегрируемость автономной системы (9) в терминах абелевых функций на римановой кривой. Таким образом, устанавливается прямая связь меж ду изомонодромными и изоспектральными системами.

Шестое уравнение Пенлеве является частным случаем уравнения Шлезингера, что позволяет интерпретировать в асимптотическим смысле связь самого PVI (уравнение изомонодромных деформаций) с его автономным аналогом - уравнением изоспектральных деформаций (9), явно интегрируемым в терминах нелинейных спецфункций. В следующем разделе мы рассмотрим основанный на этих соображениях подход, общий для всех шести уравнений Пенлеве.

\section{3. МЕТОД УСРЕДНЕНИЯ ДЛЯ УРАВНЕНИЙ ПЕНЛЕВЕ}

Уравнения Пенлеве являются интегрируемыми, и для них существуют коммутативные матричные представления (пары Лакса). Имеются шесть матричных уравнений

$$
D_{z} L_{j}-D_{x} A_{j}+\left[L_{j}, A_{j}\right]=0, \quad j=1,2, \ldots, 6
$$

где $D_{x}=d / d x ; L_{j}=L_{j}\left(y, y^{\prime}, x, z\right), A_{j}=A_{j}\left(y, y^{\prime}, x, z\right)-(2 \times 2)$-матрицы, рациональным образом зависяшие от спектрального параметра $z$, и $j$-е уравнение Пенлеве $y^{\prime \prime}-P_{j}\left(y, y^{\prime}, x\right)=0$ эквивалентно (10). Матрищы $L_{j}, A_{j}$ приведены в статье [4].

Целью данной работы является исследование асимптотического поведения шестого трансцендента Пенлеве с помощью метода усреднения Уизема, идейно сходного с упомянутым в предыдущем разделе наблюдением Гарнье. Метод применим ко всем уравнениям Пенлеве, однако из-за громоздкости вычислений для случая шестого уравнения PVI целесообразно основные положения метода проиллюстрировать на технически простейшем первом уравнении PI.

Матрищы $L_{1}$ и $A_{1}$ выглядят следующим образом:

$$
L_{1}=\left(\begin{array}{cc}
0 & 1 \\
y-z & 0
\end{array}\right), \quad A_{1}=\left(\begin{array}{cc}
-y^{\prime} & 2 y+4 z \\
-x-y^{2}+2 y z-4 z^{2} & y^{\prime}
\end{array}\right)
$$


Введем новую переменную $X$ и формально заменим все переменные $x$, явным образом входящие в формулу (11), на $X: L_{j}=L_{j}\left(y, y^{\prime}, X, z\right), A_{j}=A_{j}\left(y, y^{\prime}, X, z\right)$. Для таких матриц получаем следующую лемму.

Лемма 1. Пусть є-некоторое положительное число. Тогда система

$$
\epsilon D_{z} L_{j}-D_{x} A_{j}+\left[L_{j}, A_{j}\right]=0
$$

эквивалентна системе

$$
D_{x} X=\epsilon, \quad y^{\prime \prime}-P_{j}\left(y, y^{\prime}, X\right)=0 .
$$

ДокАЗАТЕЛЬСтво получается путем прямого вычисления. Так, для РІ система (13) принимает вид

$$
D_{x} X=\epsilon, \quad y^{\prime \prime}-3 y^{2}-X=0 .
$$

Вычисления для остальных уравнений Пенлеве принципиально аналогичны и были проведены в работе [5].

Лемма 2. Решение уравнения (12) при $\epsilon=0$ и $X=$ const представлено следующей формулой:

$$
y_{0}(x)=f_{j}(\tau+\Phi ; \vec{a}), \quad j=1,2, \ldots, 6,
$$

где $\tau=x U, \quad U=U(\vec{a}) ; f_{j}$ - периодические функиии, представимые явно в терминах әллиптических функиий Вейеритрасса или Якоби для каждого из иести уравнений Пенлеве. Вектор $\vec{a}(X)$ состоит из параметров, определяющих әллиптическую функиию $f_{j}, \Phi-$ некоторый сдвиг фазы.

ДокАЗАТЕЛЬство использует второе уравнение системы (13), где величина $X$ принимает постоянное значение. Для первого уравнения Пенлеве функция $f_{1}$ - это юфункция Вейерштрасса:

$$
f_{1}=2 \wp\left(x+\Phi ; g_{2}, g_{3}\right), \quad g_{2}=-X, \quad g_{3}=-\frac{F_{1}}{4},
$$

где $F_{1}$ - некоторый параметр. Формула (15) впервые была приведена в статье [6].

Теперь предположим, что величина $\epsilon$ положительна и мала. Мы будем искать решения уравнения (12) в виде формальных рядов по малому параметру $\epsilon$ :

$$
y(x)=y_{0}(x)+\epsilon y_{1}(x)+\cdots .
$$

Параметры, определяющие эллиптическую функцию $y_{0}=f_{j}$, подчиняются нелинейному обыкновенному дифференциальному уравнению, обычно называемому уравнением Уизема, или уравнением модуляций. Таким образом, мы ищем главньй член ряда (16) в следующей форме:

$$
y_{0}(\tau, X)=f_{j}\left(\epsilon^{-1} S(X)+\Phi(X) ; \vec{a}(X)\right), \quad D_{X} S=U
$$


ЛЕмма 3. Уравнение Уизема может быть записано следующим образом:

$$
D_{X} \operatorname{det} A_{j}=\overline{a_{22} D_{z} l_{11}}+\overline{a_{11} D_{z} l_{22}}-\overline{a_{12} D_{z} l_{21}}-\overline{a_{21} D_{z} l_{12}},
$$

где $A_{j}=\left(a_{m n}\right), L_{j}=\left(l_{m n}\right), \quad m, n=1,2$, черта сверху означает усреднение по периоду әллиптической функиии (14).

ДокАзАтельство. Легко убедиться, что уравнение (12) при $\epsilon=0$ означает независимость спектральных характеристик матрицы $A_{j}$ от переменной $x$, и выполняется условие $D_{x} \operatorname{det} A_{j}=0$. Введение формальной переменной $X$ приводит к замене правила дифференцирования: $D_{x} \rightarrow U D_{\tau}+\epsilon D_{X}$, где параметр $\epsilon$ считается малым. Далее, условие (12) дает уравнение

$$
a_{n, m}^{\prime}=\epsilon D_{z} l_{n, m}+\left[L_{j}, A_{j}\right]_{n, m}, \quad n, m=1,2 .
$$

Подставив его в равенство

$$
D_{x} \operatorname{det} A_{j}=a_{11}^{\prime} a_{22}+a_{22}^{\prime} a_{11}-a_{12}^{\prime} a_{21}-a_{21}^{\prime} a_{12},
$$

мы заменяем правило дифференцирования и получаем соотношение

$$
\left(U D_{\tau}+\epsilon D_{X}\right) \operatorname{det} A_{j}=\epsilon\left(a_{22} D_{z} l_{11}+a_{11} D_{z} l_{22}-a_{12} D_{z} l_{21}-a_{21} D_{z} l_{12}\right)+O\left(\epsilon^{2}\right) .
$$

Его усреднение, т.е. интегрирование по периоду (по “быстрой” переменной $\tau$ ), уничтожает полные производные по $\tau$, что заканчивает доказательство.

ЗАмечАниЕ 1 . Существует единственньй коэффициент многочлена $\operatorname{det} A_{j}$, чья динамика по $X$ в силу уравнения модуляций нетривиальна. Обозначим этот коэффициент через $F_{j}$. Таким образом, система Уизема может быть записана в виде одного дифференциального уравнения на $F_{j}$.

Истинность замечания может быть проверена с помощью прямых вычислений для всех шести уравнений Пенлеве. Так, для PI имеем

$$
\operatorname{det} A_{1}=16 z^{3}+4 X z-F_{1},
$$

где $F_{1}=\left(y^{\prime}\right)^{2}-2 y^{3}-2 y X$. Уравнение модуляций (17) принимает вид

$$
D_{X} \operatorname{det} A_{1}=4 z+2 \bar{y}
$$

и переписывается как $D_{X} F_{1}=-2 \bar{y}$. Принимая во внимание решение $(15)$, получаем

$$
D_{X} F_{1}=-\frac{2 \eta}{\omega}=2 e_{1}+\frac{2\left(e_{3}-e_{1}\right) E}{K},
$$

где $E=E(k), K=K(k)$ - полные эллиптические интегралы:

$$
K=\int_{0}^{1} \frac{d z}{\sqrt{\left(1-z^{2}\right)\left(1-k^{2} z^{2}\right)}}, \quad E=\int_{0}^{1} \sqrt{\frac{1-k^{2} z^{2}}{1-z^{2}}} d z, \quad k^{2}=\frac{e_{2}-e_{3}}{e_{1}-e_{3}},
$$

$e_{1,2,3}$ - корни многочлена Вейерштрасса $R_{3}(t)=4 t^{3}-g_{2} t-g_{3} ; g_{2}=-X, g_{3}=-F_{1} / 4$. 
ЗАмЕчАНИЕ 2 . Простейшим способом получения эллиптического анзаца $f_{j}$ является решение уравнения

$$
F_{j}=\text { const }_{1}, \quad X=\text { const }_{2} .
$$

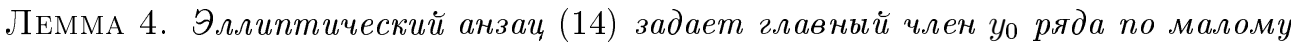
параметру є (16) для решения системы (12).

Для доказательства леммы нужно убедиться в том, что возмушение решения системы (12) при $\epsilon=0$ происходит непрерывно, по мере того как $\epsilon$ приобретает малое ненулевое значение. Соответствуюшие элементарные вычисления проиллюстрированы ниже на простейшем примере PI. Так, для $\epsilon>0$ система (12) есть $y^{\prime \prime}=3 y^{2}+X_{0}+\epsilon x$, где $X_{0}-$ константа. С помощью простых преобразований это уравнение может быть приведено к следуюшему виду:

$$
2 d x=\frac{d y}{\sqrt{2 y^{3}+X_{0}+\text { const }}}+O(\epsilon)
$$

т.е. главный член ряда (16) представлен функцией $f_{1}$ (см. (15)) при условии, что $x$ не лежит в малых окрестностях сингулярностей эллиптической функции $f_{1}$.

Теперь мы можем доказать следуюшую теорему.

ТЕОРема 1. Функиия $y_{0}$, определенная формулами (14) и (17), дает главный член асимптотического ряда для решения соответствующего уравнения Пенлеве при устремлении $|x|$ в бесконечность.

Доказательство. Масштабное преобразование $x \rightarrow \epsilon x$ ведет к замене $D_{x} X=\epsilon \mapsto$ $D_{x} X=1$ в формуле (13). Поэтому разложение (16) по малому параметру $\epsilon$ переходит в ряд по отрицательным степеням большой переменной $x$.

\section{PVI И МЕТОД УСРЕДНЕНИЯ УИЗЕМА}

Шестое уравнение Пенлеве

$$
\begin{aligned}
y^{\prime \prime}= & \frac{1}{2}\left(\frac{1}{y}+\frac{1}{y-1}+\frac{1}{y-x}\right)\left(y^{\prime}\right)^{2}-\left(\frac{1}{x}+\frac{1}{x-1}+\frac{1}{y-x}\right) y^{\prime}+ \\
& +\frac{y(y-1)(y-x)}{x^{2}(x-1)^{2}}\left(\alpha+\beta \frac{x}{y^{2}}+\gamma \frac{x-1}{(y-1)^{2}}+\delta \frac{x(x-1)}{(y-x)^{2}}\right)
\end{aligned}
$$

где греческими буквами обозначены свободные параметры, может быть получено как условие совместности следующей системы линейных уравнений:

$$
D_{z} Y=A_{6}(z, x) Y(z, x), \quad D_{x} Y=L_{6}(z, x) Y(z, x),
$$


здесь

$$
\begin{gathered}
A_{6}(z, x)=\left(\begin{array}{cc}
a_{11}(z, x) & a_{12}(z, x) \\
a_{21}(z, x) & a_{22}(z, x)
\end{array}\right)=\frac{A^{0}}{z}+\frac{A^{1}}{z-1}+\frac{A^{x}}{z-x}, \\
A^{i}=\left(\begin{array}{cc}
u_{i}+\theta_{i} & -\omega_{i} u_{i} \\
\omega_{i}^{-1}\left(u_{i}+\theta_{i}\right) & -u_{i}
\end{array}\right), \quad i=0,1, x, \quad L_{6}(z, x)=-A^{i} \frac{1}{z-x} .
\end{gathered}
$$

Положим

$$
\begin{gathered}
A^{\infty}=-\left(A^{0}+A^{1}+A^{x}\right)=\left(\begin{array}{cc}
k_{1} & 0 \\
0 & k_{2}
\end{array}\right), \\
k_{1}+k_{2}=-\left(\theta_{0}+\theta_{1}+\theta_{x}\right), \quad k_{1}-k_{2}=\theta_{\infty}, \\
a_{12}(z)=-\frac{\omega_{0} u_{0}}{z}-\frac{\omega_{1} u_{1}}{z-1}-\frac{\omega_{x} u_{x}}{z-x}=\frac{k(z-y)}{z(z-1)(z-x)}, \\
u=a_{11}(y)=\frac{u_{0}+\theta_{0}}{y}+\frac{u_{1}+\theta_{1}}{y-1}+\frac{u_{x}+\theta_{x}}{y-x}, \\
\hat{u}=-a_{22}(y)=u-\frac{\theta_{0}}{y}-\frac{\theta_{1}}{y-1}-\frac{\theta_{x}}{y-x} .
\end{gathered}
$$

Тогда

$$
\begin{gathered}
u_{0}+u_{1}+u_{x}=k_{2}, \quad \omega_{0} u_{0}+\omega_{1} u_{1}+\omega_{x} u_{x}=0, \\
\frac{u_{0}+\theta_{0}}{\omega_{0}}+\frac{u_{1}+\theta_{1}}{\omega_{1}}+\frac{u_{x}+\theta_{x}}{\omega_{x}}=0, \\
(x+1) \omega_{0} u_{0}+x \omega_{1} u_{1}+\omega_{x} u_{x}=k, \quad x \omega_{0} u_{0}=k(x) y .
\end{gathered}
$$

Решение имеет следующий вид:

$$
\begin{gathered}
\omega_{0}=\frac{k y}{x u_{0}}, \quad \omega_{1}=-\frac{k(y-1)}{(x-1) u_{1}}, \quad \omega_{x}=\frac{k(y-x)}{x(x-1) u_{x}}, \\
u_{0}=\frac{y}{x \theta_{\infty}} S_{0}
\end{gathered}
$$

где

$$
\begin{gathered}
S_{0}=y(y-1)(y-x) \hat{u}^{2}+\left[\theta_{1}(y-x)+x \theta_{x}(y-1)-2 k_{2}(y-1)(y-x)\right] \hat{u}+ \\
+k_{2}^{2}(y-x-1)-k_{2}\left(\theta_{1}+x \theta_{x}\right) \\
u_{1}=-\frac{y-1}{(x-1) \theta_{\infty}} S_{1} .
\end{gathered}
$$

Здесь

$$
\begin{gathered}
S_{1}=y(y-1)(y-x) \hat{u}^{2}+\left[\left(\theta_{1}+\theta_{\infty}\right)(y-x)+x \theta_{x}(y-1)-2 k_{2}(y-1)(y-x)\right] \hat{u}+ \\
+k_{2}^{2}(y-x)-k_{2}\left(\theta_{1}+x \theta_{x}\right)-k_{1} k_{2} \\
u_{x}=\frac{y-x}{x(x-1) \theta_{\infty}} S_{\infty}
\end{gathered}
$$


где

$$
\begin{aligned}
S_{\infty}= & y(y-1)(y-x) \hat{u}^{2}+\left[\theta_{1}(y-x)+x\left(\theta_{x}+\theta_{\infty}\right)(y-1)-2 k_{2}(y-1)(y-x)\right] \hat{u}+ \\
& +k_{2}^{2}(y-1)-k_{2}\left(\theta_{1}+x \theta_{x}\right)-x k_{1} k_{2} .
\end{aligned}
$$

Условие совместности для системы (20) предполагает

$$
y^{\prime}=\frac{y(y-1)(y-x)}{x(x-1)}\left(2 u-\frac{\theta_{0}}{y}-\frac{\theta_{1}}{y-1}-\frac{\theta_{x}-1}{y-x}\right) .
$$

Таким образом, у удовлетворяет PVI с параметрами

$$
\alpha=\frac{1}{2}\left(\theta_{\infty}-1\right)^{2}, \quad \beta=-\frac{1}{2} \theta_{0}^{2}, \quad \gamma=\frac{1}{2} \theta_{1}^{2}, \quad \delta=\frac{1}{2}\left(1-\theta_{x}^{2}\right) .
$$

Теперь мы применим идеи, изложенные в предыдущем разделе, к асимптотическому анализу свойств шестого трансцендента Пенлеве. Сначала вычислим определитель матрицы $A_{6}$. Используя формулы $(22),(23)$, получим

$$
a_{11}(z)=-R^{-1}(z) S
$$

где

$$
\begin{gathered}
S=k_{1} z^{2}+z\left[x\left(u_{0}+u_{1}+\theta_{0}+\theta_{1}\right)+u_{0}+\theta_{0}+u_{x}+\theta_{x}\right]+O\left(z^{0}\right), \\
a_{22}(z)=-R^{-1}(z)\left\{k_{2} z^{2}-z\left[(x+1) u_{0}+x u_{1}+u_{x}\right]+O\left(z^{0}\right)\right\},
\end{gathered}
$$

$R(t)=t(t-1)(t-x) ; O\left(z^{j}\right)$ означает степени $z$ порядка не выше чем $j$. Матричные элементы $a_{12}, a_{21}$ дают члены меньшего порядка по $z$, поэтому их можно игнорировать при вычислении двух старших членов многочлена $\operatorname{det} A_{6}$. Таким образом получаем

$$
\operatorname{det} A_{6}=R^{-2}(z) S,
$$

где

$$
\begin{aligned}
S= & k_{1} k_{2} z^{4}-z^{3}\left[k_{1}\left(u_{0}(x+1)+u_{1} x+u_{x}\right)-\right. \\
& \left.-k_{2}\left(x\left(u_{0}+u_{1}+\theta_{0}+\theta_{1}\right)+u_{0}+\theta_{0}+u_{x}+\theta_{x}\right)\right]+O\left(z^{2}\right) .
\end{aligned}
$$

Полагая

$$
\operatorname{det} A_{6}=R^{-2}(z)\left[k_{1} k_{2} z^{4}+F_{6} z^{3}+O\left(z^{2}\right)\right],
$$

найдем коэффициенты $F_{6}$, определяющие уиземову динамику:

$$
F_{6}=\left(k_{1}-k_{2}\right)\left(u_{1}+x u_{x}\right)-x\left(2 k_{1} k_{2}+\theta_{x}\right)-2 k_{1} k_{2}-k_{2} \theta_{1} .
$$

Теперь нужно вывести условие на эллиптическую функцию из уравнения (26). Используя (23), получим соотношение

$$
\begin{aligned}
\theta_{\infty}\left(u_{1}+x u_{x}\right)= & -R(y) \hat{u}^{2}+\hat{u}\left[(y-1)(y-x)\left(2 k_{2}+\theta_{\infty}\right)-\right. \\
& \left.-\theta_{1}(y-x)-x \theta_{x}(y-1)\right]+k_{1} k_{2}(x+1-y)+k_{2}\left(\theta_{1}+x \theta_{x}\right),
\end{aligned}
$$


которое с учетом (24) и (22) переходит в следуюшее:

$$
\begin{aligned}
\theta_{\infty} & \left(u_{1}+x u_{x}\right)=-\frac{x^{2}(x-1)^{2}}{4 R(y)}\left(y^{\prime}\right)^{2}+ \\
& +\frac{1}{2} y^{\prime} x(x-1)\left(B+\frac{1}{R(y)}\left[(y-1)(y-x)\left(2 k_{2}+\theta_{\infty}\right)-\theta_{1}(y-x)-x \theta_{x}(y-1)\right]\right)- \\
& -\frac{1}{4} R(y) B^{2}+\frac{1}{2} B\left[x \theta_{x}(y-1)-(y-1)(y-x)\left(2 k_{2}+\theta_{\infty}\right)+\theta_{1}(y-x)\right]+ \\
& +k_{1} k_{2}(x-y+1)+k_{2}\left(\theta_{1}+x \theta_{x}\right),
\end{aligned}
$$

где

$$
B=\frac{\theta_{0}}{y}+\frac{\theta_{1}}{y-1}+\frac{\theta_{x}+1}{y-x} .
$$

Подставив полученное выражение в (26), находим окончательное условие на риманову поверхность $\left(y^{\prime}, y\right)$ рода 1 и соответствуюшую эллиптическую униформизацию (считаем $x$ и $F_{6}$ параметрами):

$$
\begin{aligned}
& x^{2}(x-1)^{2}\left(y^{\prime}\right)^{2}-2 y^{\prime} x(x-1) y(y-1)+y^{4}\left[1-\left(k_{1}-k_{2}\right)^{2}\right]+ \\
& \quad+2 y^{3}\left[\left(k_{1}+k_{2}\right) C-1+2 x \theta_{x}\left(1-k_{2}\right)+2 F_{6}\right]-y^{2} S+ \\
& \quad+2 y x\left[2 k_{1} k_{2}(x+1)+2 x \theta_{x}\left(1-k_{2}\right)+2 F_{6}-\theta_{0} C\right]-x^{2} \theta_{0}^{2}=0,
\end{aligned}
$$

где

$$
\begin{gathered}
C=(x+1)\left(k_{1}+k_{2}\right)+x \theta_{x}+\theta_{1}, \\
S=C^{2}-1-2 x \theta_{0}\left(k_{1}+k_{2}\right)+4 k_{1} k_{2}\left(x^{2}+x+1\right)+4 x(x+1)\left(1-k_{2}\right) \theta_{x}+4(x+1) F_{6} .
\end{gathered}
$$

Чтобы начать процедуру уиземова асимптотического исследования, необходимо вывести уравнение модуляций в дополнение к анзацу (27). Для этого заменим переменные $x$ на $X$ в формуле (25) и продифференцируем ее по $X$ :

$$
D_{X} \operatorname{det} A_{6}=\frac{z^{4}}{(z-X) R^{2}(z)}\left(2 k_{1} k_{2}+D_{X} F_{6}\right)+O\left(z^{3}\right) .
$$

Воспользуемся условием (17). Итак, выполняется

$$
D_{z} L_{6}(z, x)=\frac{A_{x}}{(z-X)^{2}}, \quad D_{z} l_{22}=-\frac{u_{x}}{(z-X)^{2}},
$$

откуда следуют соотношения

$$
\begin{aligned}
a_{11} D_{z} l_{22} & =\frac{u_{x}}{R(z)(z-X)^{2}}\left[z^{2} k_{1}+O(z)\right], \\
a_{22} D_{z} l_{11} & =-\frac{u_{x}+\theta_{x}}{R(z)(z-X)^{2}}\left[z^{2} k_{2}+O(z)\right],
\end{aligned}
$$

подставляя которые в (17), получаем

$$
D_{X} \operatorname{det} A_{6}=\frac{z^{2}\left[\bar{u}_{x}\left(k_{1}-k_{2}\right)-k_{2} \theta_{x}\right]+O(z)}{R(z)(z-X)^{2}},
$$


где черта сверху означает усреднение. Сравнение формул (28) и (29) дает уравнение модуляций:

$$
D_{X} F_{6}=\bar{u}_{x}\left(k_{1}-k_{2}\right)-k_{2} \theta_{x}-2 k_{1} k_{2} .
$$

Перепишем (30) в исходных координатах $y, X$. Для этого используем равенство

$$
u_{x}\left(k_{1}-k_{2}\right)=\frac{y-X}{X(X-1)} S,
$$

где

$$
\begin{aligned}
S= & R(y) \hat{u}^{2}+\hat{u}\left[\theta_{1}(y-X)+X\left(\theta_{x}+\theta_{\infty}\right)(y-1)-2 k_{2}(y-1)(y-X)\right]+ \\
& +k_{2}^{2}(y-1)-k_{2}\left(\theta_{1}+X \theta_{x}\right)-X k_{1} k_{2} .
\end{aligned}
$$

Подставим (31) в (30), снова используем (22)-(24) и после упрошения получаем уравнение модуляций в виде

$$
\begin{aligned}
D_{X} F_{6}= & \frac{1}{2}\left(k_{1}-k_{2}\right) D_{X} \bar{y}+\frac{\left(k_{2}-k_{1}\right)\left(k_{2}-k_{1}+1\right)}{2 X(X-1)} \overline{y^{2}}+\frac{\bar{y}}{X(X-1)} S+ \\
& +\frac{1}{2(X-1)}\left[\theta_{0}\left(k_{2}-k_{1}\right)+2 X\left(2 k_{1} k_{2}+\theta_{x}\right)+2 k_{2}\left(k_{1}+k_{2}+\theta_{1}\right)+2 F_{6}\right]- \\
& -k_{2} \theta_{x}-2 k_{1} k_{2},
\end{aligned}
$$

где

$$
\begin{aligned}
S= & \frac{1}{2}\left(k_{2}-k_{1}\right)\left[X\left(k_{2}-k_{1}-\theta_{x}\right)+\theta_{0}+\theta_{x}+1\right]- \\
& -X\left(2 k_{1} k_{2}+\theta_{x}\right)-k_{2}\left(k_{1}+k_{2}+\theta_{1}\right)-F_{6} .
\end{aligned}
$$

Здесь $\bar{y}$ обозначает среднее эллиптической функции $y$, задаваемой уравнением $(27), F_{6}$ и $X$ (вместо $x)$ считаются параметрами.

\section{5. ЧАСТНЫЕ РЕШЕНИЯ УРАВНЕНИЯ МОДУЛЯЦИЙ И РVІ}

Исследование систем (27), (32) в общем виде затруднительно, более того, остается открытым вопрос о фазовом сдвиге $\Phi$ в эллиптическом анзаце (14). Поэтому мы остановимся на простейшем решении, соответствуюшем сильно вырожденной кривой (27). Если искать частные решения (27)-(32) в классе элементарных функций, то нетрудно отметить наличие асимптотической однородности формулы (27) для больших $x$. Обозначим $y=x \xi$ и перепишем (27) в переменных $x \xi^{\prime}$ и $\xi$. Дискриминант этого многочлена выглядит следующим образом:

$$
\begin{aligned}
D= & \xi^{4}\left(k_{2}-k_{1}\right)^{2}-2 \xi^{3}\left[\left(k_{2}+k_{1}\right)^{2}+2 \theta_{x}\left(1-k_{2}\right)+2 F_{6} x^{-1}+O\left(x^{-1}\right)\right]+ \\
& +\xi^{2}\left[\left(k_{2}+k_{1}\right)^{2}+4 k_{1} k_{2}+4 \theta_{x}\left(1-k_{2}\right)+4 F_{6} x^{-1}+O\left(x^{-1}\right)\right]- \\
& -2 \xi\left[2 F_{6} x^{-2}+O\left(x^{-2}\right)\right]+O\left(x^{-2}\right) .
\end{aligned}
$$


Рассмотрим условие сильного вырож дения для римановой кривой (27). Многочлен (33) имеет корни, асимптотически сливающиеся в два двойных корня, если

$$
\begin{gathered}
\theta_{x}=0 \quad F_{6}=-2 k_{1} k_{2} X+o(X), \quad X \rightarrow \infty, \\
D=\xi^{2}(\xi-1)^{2}\left(k_{2}-k_{1}\right)^{2}+O\left(X^{-1}\right) .
\end{gathered}
$$

Этот случай мы назовем двойным или сильным вырождением. Подставляя условие (34) в (27), легко получаем соответствуюшую асимптотику для решения: $\xi=1+o(1)$ и, следовательно,

$$
y=x+o(x), \quad x \rightarrow \infty,
$$

где $o(x)$ обозначает члены, растущие не быстрее чем $\ln x$. Нетрудно также убедиться, что решение (34), (35) удовлетворяет уравнению модуляций (32).

Таким образом, мы приходим к следуюшей теореме.

Teоpema 2. В случае $\theta_{x}=0 \quad(\delta=1 / 2)$ шестое уравнение Пенлеве имеет решение с асимптотикой $(35)^{1)}$.

Для доказательства теоремы в дополнение к упомянутому выше следует отметить, что сильное вырождение эллиптического анзаца (27) преобразует фазовый сдвиг $\Phi$ в формуле (14) в сдвиг по переменной $x$, который может быть найден при помощи простой итеративной процедуры, вычисляюшей члены разложения (35).

Благодарности. Работа выполнена при поддержке грантов INTAS № 99-01782 и РФФИ № 99-0100656.

\section{Список литературы}

[1] P. Painlevé. Acta Math. 1902. V. 25. P. 1-86.

[2] L. Schlesinger. J. fur Math. 1912. V. 141. P. 96-145.

[3] R.Garnier. Ann. Sci. École Norm. Super. (3). 1917. V. 34. P. 239-353.

[4] M. Jimbo, T. Miwa. Phys. D. 1981. V. 3. P. 407-448.

[5] В. Л. Верещагин. Дифф. уравнения. 1999. Т. 35. Вып. 6. С. 837-839.

[6] С. П. Новиков. Функц. анализ и его прилож. 1990. Т. 24. Вып. 4. С. 43-53.

[7] M. Mazzocco. Picard and Chazy solutions to the Painlevé VI equation. math.AG/9901054.

Поступила в редакцию 29.XII.2000 г.

\footnotetext{
1) Частный случай этого решения для PVI при условии $\alpha=(2 \mu-1) / 2, \beta=\gamma=0, \delta=1 / 2$ и полуцелого $\mu$ был описан в работе [7]
} 